\title{
Incidence rate and financial burden of medical errors and policy interventions to address them: a multi-method study protocol
}

\author{
Ehsan Ahsani-Estahbanati ${ }^{1}$ - Leila Doshmangir ${ }^{1,2}$ (D) $\cdot$ Behzad Najafi $^{3} \cdot$ Ali Akbari Sari $^{4}$. \\ Vladimir Sergeevich Gordeev ${ }^{5,6}$
}

Received: 30 March 2021 / Revised: 17 September 2021 / Accepted: 1 October 2021 /

Published online: 5 November 2021

(C) The Author(s) 2021

\begin{abstract}
Medical error is one of the most critical challenges facing medical services. They pose a substantial threat to patient safety, and their costs draw attention from policymakers, health care planners and researchers. We aim to make a realistic estimation of medical error incidence and related costs and identify factors influencing this incidence in Iranian hospitals. In the first phase of this multi-method study, through two reviews of systematic reviews and a meta-analysis, we will estimate the incidence of medical errors and the strategies to reduce them. We will extract available data among 41 hospitals supervised by the East Azerbaijan University in the second phase. We will also develop a model and use a Delphi method to predict medical errors incidence and calibrate our model output using the Monte Carlo simulation. We will compare this estimation with the incidence rate based on meta-analysis results from the first phase. In the third phase, we will investigate the relationship between several factors potentially influencing medical error incidence. In the fourth phase, we will estimate costs associated with medical errors by conducting a patient records review and matching those with claims related to medical errors. In the fifth phase, we will present a policy brief related to strategies for medical errors and associated costs reduction in Iran. Our findings could benefit Iranian and policymakers in other countries to reduce medical errors and associated costs.
\end{abstract}

Keywords Medical error · Incidence · Financial burden · Policy intervention · Global health policy and systems research
Abbreviations
WHA72 The 72nd world health assembly
CDI Clostridium difficile infection
CMA Comprehensive meta-analysis
MoHME Ministry of health and medical education
CI Confidence interval

Leila Doshmangir

doshmangirl@tbzmed.ac.ir

Extended author information available on the last page of the article 


\section{Background}

A medical error is a "failure of a planned action to be completed as intended or the use of a wrong plan to achieve an aim" (Institute of Medicine Committee on Quality of Health Care in, 2000). It is a significant source of preventable morbidity and mortality among patients. Medical errors also increase the overall costs of hospitalisation and decrease the quality of the healthcare system. Hospitals' most common medical errors are administering the wrong medication, inappropriate blood transfusion, wrong-site surgery, under and overtreatment, suicide, falls, improper patient identification, and misdiagnosis (Carver and Hipskind, 2019). Severe consequences of medical errors frequently occur in intensive care units, operating rooms and emergency departments. Medical errors are associated with patient's age, new procedures, urgency and the severity of the medical services (Carver and Hipskind, 2019). In May 2019, the 72nd World Health Assembly (WHA72) endorsed the establishment of World Patient Safety Day to raise global awareness about patient safety and encourage global solidarity and action. During WHA72, patient safety was crucial for countries to progress towards Universal Health Coverage, as extending health care should mean extending safe care. It called for patient safety to become a global health priority (Ghebreyesus, 2019).

Previous studies have shown that the global burden associated with medical errors is substantial, irrespective of the country's income level. For example, medical errors are the third leading cause of death in the United States, with more than 250,000 annual deaths in the United States attributed to medical errors (Makary and Daniel, 2016). Based on a systematic review, medication errors in hospitalised patients in Middle Eastern countries range from 11 to $90 \%$ (Thomas et al., 2016). As one of the main safety problems, medical errors impose a considerable financial burden on the health systems. Given the preventable nature of most medical errors, health policymakers and healthcare professionals should consider the associated financial dimension (Andel et al., 2012). The annual expenditure related to medical errors is approximately $\$ 20$ billion in the United States, with half of the claims related to outpatient care (Rodziewicz and Hipskind, 2019). The annual cost of measurable medical errors that harm patients was \$17.1 billion in the US in 2008 (Van Den Bos et al., 2011). Moreover, the annual avoidable costs of nosocomial infections in the US ranged from $\$ 142$ million to $\$ 4.25$ billion in 2016 (Schmier et al., 2016). In Europe, the thirty-day mortality in patients with healthcare facility-acquired nosocomial Clostridium difficile infection (CDI) varied from 3\% (France) to $30 \%$ (UK), the proportion of total mortality attributable to CDI ranged from 0 to $70 \%$ (primarily due to difference in the definition), and CDI recurrence rates ranged from 1 France to 35\% (Ireland). The incremental costs of CDI ranged from $£ 4,577$ (Ireland) to $£ 8,843$ (Germany) (standardised to 2010 GBP) (Wiegand et al., 2012). Another study showed that the mean cost per error per study ranged from $€ 2.58$ to $€ 111,7$ thousand, with a difference of greater than $€ 100$ thousand per individual medication error (Walsh et al., 2017).

The Iranian health care system is no exception. A systematic review and meta-analysis showed that the prevalence rate of medical errors in Iran ranges from 0.06 to $42 \%$ (2020) (Mosadeghrad et al., 2020). 44\% of nurses in the paediatric ward in hospitals in Yazd reported making medical errors once or twice over the six months in 2015 (Salmani and Fallah Tafti, 2016). In a case study conducted in one of the hospitals of West Azerbaijan province, the rate of medication errors was 58\% (Gahremani and Sharifi, 2016). To the best of our knowledge, no previous study estimated costs associated with medical errors in Iran. Therefore, an accurate estimation of the incidence and the economic burden associated 
with medical errors is necessary for developing and implementing interventions focusing on their reduction. Hence, this study will aim to estimate the incidence of medical errors in hospitals in Iran. We will explore which factors (i.e., hospital types, seasonality, work shifts, medical error types) affect the medical error intensity. We will also examine associated costs and develop a model/formula to estimate medical error incidence globally and locally. We will select viable, evidence-based policy interventions to reduce medical errors that could be recommended to policy-makers in Iran and other countries.

\section{Methods}

This study is a multi-method study and will be conducted in five phases.

\subsection{Phase 1}

We will pursue two objectives in this phase. The first objective is to find evidence-based ways to reduce medical errors and their costs that will feed into analysis planned for Phase 5 , using a systematic review of systematic reviews. The second objective is to estimate the international rates of medical errors through a meta-analysis, and these results will be used in the fifth step of Phase 2.

The search will be conducted using several databases (i.e., PubMed, Scopus, Ovid Medline and Embase). Additionally, we will supplement this review with a manual check for all relevant references in included studies. Our search strategy will be similar for both steps and will be adjusted for each database. For example, we plan to use the following combination for search in the PubMed database: $\left(_{(()(()(()(()((\text {medical errors[MeSH }}\right.$ Terms] OR "recording error:[Title/Abstract]) OR "no harm"[Title/Abstract] OR "hospital infection"[Title/Abstract]) OR "healthcare associated infections :[Title/Abstract]) OR "transfusion error”[Title/Abstract]) OR “prescription error”[Title/Abstract]) OR “prescribing error"[Title/Abstract]) OR “CPR error"[Title/Abstract]))) OR “medication error"[Title/ Abstract]) OR “near miss”[Title/Abstract]) OR “equipment error”[Title/Abstract]) OR "complication*”[Title/Abstract])AND systematic[sb]).

After removing duplicate references, two reviewers will independently assess all the titles and abstracts to determine whether a full-text review is required. A third independent reviewer will resolve any disagreements between the two reviewers. Our proposed inclusion criteria: (a) systematic reviews or meta-analysis studies, (b) studies presenting solutions regarding medical error reduction or medical error costs (in the first step), and (c) studies focusing on medical error incidence or prevalence rate (in the second step), which will enter the study regardless of whether these reviews were based on reported errors, an examination of medical profiles, observational studies or other methods. Our exclusion criteria will be: (a) studies published in languages other than English; (b) studies about a medical error that were conducted in settings other than the hospital; and (c) studies focusing only on a particular group of employees where generalizability to other groups is not feasible (i.e., only nurses, physicians, pharmacists). AMSTAR checklist for systematic reviews will be used for the quality appraisal (Shea et al., 2017). Two researchers will appraise the quality independently. The reviews risk of bias will be assessed using the Cochrane method (Higgins and Altman, 2008). Reviews with the lowest score will be deleted.

We will extract studies information (author, the publication date, study objectives, number of publications included in each review) in both steps, policy interventions types related 
to medical error reduction (in the first step), and incidence or prevalence rate of medical errors/overall result (in the second step). We will extract data from systematic reviews and not the primary studies included in each systematic review. Analysis of appraised reviews will be descriptive. Policy interventions related to medical errors and their cost reduction will be presented descriptively in the first step. We will use Comprehensive Meta-Analysis Software (CMA) to do a meta-analysis in the second step. We will use a Chi-squared test for homogeneity assessment and a funnel plot to assess publication bias in studies. We will pool extracted data through a fixed-effect or random-effect meta-analysis based on the heterogeneity among included studies. A random-effects model will be adopted if the presented outcomes have moderate or high statistical heterogeneity; with low statistical heterogeneity, data will be pooled using a fixed-effect model.

\subsection{Phase 2}

Phase 2 will estimate medical errors incidence across several medical error types in all 41 hospitals supervised by the Tabriz University of medical sciences in East Azerbaijan Province in Iran. The selected hospitals are exemplary of other Iranian hospitals and reflect different ownership types. All hospitals use similar, if not identical, reporting forms and processes of data collection. For instance, forms across hospitals are expected to contain information about the type of error (surgical, medication, healthcare-associated infections, diagnostic, transfusion and testing, equipment and complication), date of the error, error intensity (no harm, near miss and sentinel event), location/ward and work shift when the medical error has happened (day, evening and night). Generally, the hospital data on medical errors are collected monthly and referred to the University's Treatment Deputy, who calculates the incidence rate by dividing the number of reported medical errors by all number of inpatient admissions performed in the hospitals as a denominator.

This phase will have five steps. First, relevant data will be gathered through medical error reporting forms collected in each hospital and the University's annual reports. The data will be extracted, where available, for one year. As explained earlier, the forms are designed by the Iranian Ministry of Health and Medical Education (MoHME) and are similar across all hospitals in the country. The University's annual report includes reported medical error cases, while non-medical errors are excluded. The annual report also contains the incidence rate of medical error for each hospital type and the number of hospital inpatient admissions in a current year. In the second step, to estimate the parameters for which accurate data are not available, we will conduct a three-round classic Delphi study with key experts involved in medical errors at Tabriz University of Medical Sciences. Based on the previous research experience and six hospital categories, we will aim to recruit about 60 participants. Recruitment will use purposive sampling. The Delphi study will be used to reach a consensus regarding factors influencing the probability of reporting, over-reporting and under-reporting of medical errors, using $70 \%$ as a threshold for reaching a consensus. In the third step, we will estimate the incidence of medical errors by using a proposed formula, based on probability values estimated during the Delphi study and assuming that the median number of episodes will fix the effect of over-reporting:

$$
I=\frac{A}{(1-P n) \times P c \times \operatorname{Pr} \times P a}
$$

Note: $I$ : incidence rate of medical error; $A$ : annual reported incidence of medical error by Treatment Deputy, $(1-P n)$ : Probability of error reporting in hospitals, $P c$ : Probability of 
confirming error reported by hospitals, $\operatorname{Pr}$ : Probability of error reporting to Treatment Deputy by hospitals, $\mathrm{Pa}$ : Probability of confirming error reported by hospitals by Treatment Deputy.

We will calibrate the model output in the fourth step by simulating the random component of the model and show the probability of values using Monte Carlo simulation with at least 10,000 iterations (Buhara and Pehlivan, 2019). We will calculate means and the upper and lower ranges of outputs to enhance the accuracy of the analysis and present the 95\% confidence interval (CI) for estimates. We will estimate outcomes per each hospital type and all hospitals together, which we believe would yield results that are meaningful for interpretation in clinical practice. Data for individual hospitals will not be analysed separately. In the fifth step, we will compare our estimates with the incidence rate from the meta-analysis conducted in the first phase. We will use a two-sample Student's t-test to compare medical error incidence with the systematic-reviewed incidence rate. All quantitative analyses will be performed using Stata 11 (College Station, TX: Stata Corp LP., 2009). To confirm, the results of this phase will be checked and scrutinised by country experts.

\subsection{Phase 3}

This phase will investigate the relationship between several factors associated with the medical error intensity (no harm, near miss and sentinel event) in the studied hospitals. Explanatory factors will include seasonality, hospital types (public educational, public non-educational, private, charity, military and social security), work shifts (day, evening and night), and medical error types (surgical, medication, healthcare-associated infections, diagnostic, transfusion and testing, equipment and complication). We will gather related data by forms that were described earlier in Phase 2. We will consider using log-linear and multinomial logistic regression models. Intensity will be the primary outcome, as its interpretation would be meaningful, particularly for clinical practice, due to its possible impact on patient's health. The medical error type reflects the content of the error (e.g., surgical, drug, fall, hospital infection). It is expected to contain around 11 categories, which could potentially be grouped into six broader groups. It also identifies the area of care affected rather than the hazardous characteristics of the error.

\subsection{Phase 4}

This phase will be used to estimate the costs associated with the medical errors. Implementation of this phase in all hospitals $(n=41)$ is time-consuming, costly, and requires a lot of administrative correspondence and coordination-therefore, unfeasible. Also, we anticipate heterogeneity in the incidence rate of medical errors between hospitals (based on medical error report rates), which depends on the hospital's ownership type. Hence, we will deploy stratified random sampling to select at least one hospital in each of the six-hospital types based on their ownership. The exact sample size will be determined and adjusted across simple random sampling based on the detected incidence rate of medical errors in each hospital type in a 95\% confidence interval. Estimated costs associated with medical errors in each selected hospital type will then be generalized for all hospitals included in previous Phases. If a particular hospital refuses to participate, we will replace it with another hospital belonging to the same category. For example, if a refusing hospital in an educational hospital, we will use stratified random sampling to randomly select another hospital from the same educational hospital category to replace it. 
With the help of two expert nurses in each hospital, medical errors will be categorised based on the medical error types used in the previous phase. Since medical errors in patients cause different injuries and complications, we will differentiate complications in each error category. The nurses will determine the medical error services and separate them from routine treatment services. They will also review patients' profiles and determine what services and procedures (including tests, scans, surgery, re-admission, or increase of stay in hospitals) occurred due to medical errors. In cases when it is not possible to do so, we will consult a relevant specialist. Reviewing the patient's profile will be done confidentially. No identification characteristics for the patients, nurses and specialists will be used or recorded.

The Iranian medical tariff book will be used to calculate the costs of identified medical errors independently for each patient. All services will be categorized into two groups: with and without a medical tariff. For services without a tariff (i.e., medicine and supplies), we will use the cost of the purchase invoice with the addition of the expected profit as the basis for cost calculation. We will then aggregate these costs and calculate the average service cost per hospital group for each medical error category. We will multiply the average service cost by the frequency in each hospital group's error to calculate total services cost. To produce an overall estimate for Iran, the hospitals will be weighted based on the total incidence rate of medical errors for each hospital type and the number of hospitals included in each hospital type in Iran. We will use a cost simulation method when we do not have access to patients' medical profiles. We will use the judgment of nurses, the research team, and two or three specialists in each medical error category to determine the content of the routine therapeutic and diagnostic services necessary for each patient who faced the medical error. We will also gather data regarding patient claims and obtain related costs by the judicial votes once they are processed, and the compensation amounts are determined. In this stage, we will combine all claims costs and will not use sampling.

\subsection{Phase 5}

This phase will identify possible interventions to control and reduce the medical error incidence rates and related costs specific to the Iranian context. This phase will consist of two steps: (1) a qualitative study to determine interventions related to medical error and its cost reduction and (2) developing policy options related to medical error and its cost reduction presentation.

In the first step, we will use semi-structured interviews to find interventions to reduce medical error and associated costs relevant to the Iranian context. Semi-structured interviews will be based on systematic reviews (the first step of the first phase) and research team views. The lead researcher will interview the experts until data saturation and use the qualitative content analysis method to analyse the data (Hsieh and Shannon, 2005). We plan to purposively select 20 experts in medical error from all over the country. Should we fail to reach data saturation after conducting these 20 interviews, we will recruit more experts using the snowball technique until the data saturation is reached. We will consider inviting experts with at least five years of professional experience in medical errors or cost reduction and experts with patient safety responsibilities, hospital management experience and Treatment Deputies. All interviews will be transcribed verbatim and read several times to achieve immersion. Immersion will be achieved by carefully reading transcripts, listing meaningful, recurrent ideas and identifying critical issues in the data (Vaismoradi et al., 2016). Next, the codes will be extracted by 
reading data word by word. Codes will then be manually combined into subcategories and categories. Definitions for each category, subcategory, and code will be developed. To increase the scientific credibility of our study, we will use procedures presented by Goba and Lincoln to consider confirmability, dependability, transferability and acceptance (Strauss and Corbin, 1994). To reach these criteria, the research team and three experts outside of the team will work together. They will code a sample of interviews to reach a consensus and check data reliability. Also, we will check semantic validity to ensure that different phrases or words that are part of a category have a similar meaning, and they all belong to the same category. After completing the analyses, we will present the results.

In the second step, we will present policy interventions related to medical error and its cost reduction. This step will be based on the results of the overview of systematic reviews and interviews results. We will present a policy brief that states the problem extent, details the medical error incidence rate and outlines its consequences at local, national and international levels. The expert panel will prioritise these solutions for each medical error category. Then we will explore a maximum of three prioritised options and their executive considerations such as feasibility, cost, needed resources, quality, safety, assessment necessity, and suggested interventions monitoring. The global evidence (based on systematic reviews) will be further adjusted to the Iranian context through expert feedback and efficiency and effectiveness criteria. We will present the prioritised options, their merits and disadvantages to policymakers, who will then be able to select the best option based on context, resources, and current situations and plan, implement and evaluate related corrections.

\section{Discussion}

Despite long-term efforts to control and prevent medical errors and increase patients' safety, medical errors are still one of the leading causes of death in the world. This study will estimate the incidence rate of medical errors in Iran and worldwide, elicit factors that affect incident rates, estimate the economic burden of medical errors, and outline international and national interventions that can be used to reduce medical errors. We will present policy briefs to policymakers to draw their attention to this critical issue. Although reviews written in languages other than English could potentially add to the comprehensiveness of information and insight into the different medical errors aspects, this study will not include these reviews due to the lack of expert translators and time constraints. Nonetheless, the study will provide the foundation for future work to estimate the medical error rates nationally and internationally.

Acknowledgements This study forms PhD research project of the lead author (Ehsan Ahsani-Estahbanati), supported by the Tabriz University of Medical Sciences.

Author contributions EA-E and LD conceived the study and drafted the article. VSG is in charge of the statistical analysis, and BN will perform the economic analysis. All authors participated in the final design and revision of the manuscript.

Funding No funds were received.

Availability of data and materials The datasets used and/or analyzed during the current study will be available from the corresponding author on reasonable request. 


\section{Declarations}

Conflict of interest The authors declare that they have no competing interests.

Ethics approval and consent to participate Tabriz University of Medical Sciences, Iran, the medical ethics committee approved the study (Approval No: IR.TBZMED.REC.1398.557). There will be no patient involvement during the development of the research question, design, recruitment, and study conduct. The primary focus of the study is on healthcare experts who participate in the Delphi study and interviews. All those who will participate in interviews and the Delphi phase will give written informed consent by email or in person before recruitment. The study is non-invasive and imposes no significant risks to participants. Participants can withdraw from the study at any point.

Consent for publication Dissemination will include peer-reviewed publications and presentations at national and international conferences.

Open Access This article is licensed under a Creative Commons Attribution 4.0 International License, which permits use, sharing, adaptation, distribution and reproduction in any medium or format, as long as you give appropriate credit to the original author(s) and the source, provide a link to the Creative Commons licence, and indicate if changes were made. The images or other third party material in this article are included in the article's Creative Commons licence, unless indicated otherwise in a credit line to the material. If material is not included in the article's Creative Commons licence and your intended use is not permitted by statutory regulation or exceeds the permitted use, you will need to obtain permission directly from the copyright holder. To view a copy of this licence, visit http://creativecommons.org/licenses/by/4.0/.

\section{References}

Andel, C., Davidow, S.L., Hollander, M., Moreno, D.A.: The economics of health care quality and medical errors. J. Health Care Finance 39, 39 (2012)

Buhara, O., Pehlivan, S.: Monte Carlo simulation of reasons for early failure of implants: effects of two risk factors. Br. J. Oral. Maxillofac. Surg. 57, 12-20 (2019)

Carver N, Gupta V, Hipskind JE. Medical Error. In: StatPearls [Internet]. Treasure Island (FL): StatPearls Publishing (2021)

Gahremani, R., Sharifi, H.: Investigating the prevalence of medication errors in preparation and the administration of intravenous drugs in a second-level university hospital. Urmia Med. J. 27, 140-147 (2016)

Ghebreyesus, T.A.: Global action on patient safety [Online]. World Health Organization: World Health Organization. Available: apps.who.int/gb/ebwha/pdf_files/WHA72/A72_26-en.pdf [Accessed 12/18/2019 2019] (2019)

Higgins, J.P.T., Altman, D.G. Assessing risk of bias in included studies. In: Higgins JPT, Green S, eds. Cochrane handbook for systematic reviews of interventions. Wiley (pp. 187-241, 2008)

Hsieh, H.-F., Shannon, S.E. Three approaches to qualitative content analysis. Qual. Health Res. 15, 12771288 (2005). https://doi.org/10.1177/1049732305276687

Institute of medicine committee on quality of health care IN, A.: In: Kohn, L.T., Corrigan, J.M. \& Donaldson, M.S. (eds.) To Err is Human: building a safer health system. Washington (DC): National Academies Press (US) Copyright 2000 by the National Academy of Sciences. All rights reserved (2000)

Makary, M.A., Daniel, M.: Medical error-the third leading cause of death in the US. BMJ 353, i2139 (2016)

Mosadeghrad, A.M., Isfahani, P., Yousefinezhadi, T.: Medical errors in Iranian hospitals: systematic review. Tehran Uni. Med. J. 78, 239-247 (2020)

Rodziewicz, T.L., Hipskind, J.E.: Medical error prevention Statpearls. StatPearls Publishing LLC, Treasure Island (FL) (2019)

Salmani, N., Fallah Tafti, B.: Frequency, type and causes of medication errors in pediatric wards of hospitals in Yazd, the Central of Iran. Int. J. Pediatrics 4, 3475-3487 (2016)

Schmier, J.K., Hulme-Lowe, C.K., Semenova, S., Klenk, J.A., Deleo, P.C., Sedlak, R., Carlson, P.A.: Estimated hospital costs associated with preventable health care-associated infections if health care antiseptic products were unavailable. Clinico. Econ. Outcomes Res.: CEOR 8, 197 (2016) 
Shea, B.J., Reeves, B.C., Wells, G., Thuku, M., Hamel, C., Moran, J., Moher, D., Tugwell, P., Welch, V., Kristjansson, E.: AMSTAR 2: a critical appraisal tool for systematic reviews that include randomised or non-randomised studies of healthcare interventions, or both. BMJ (2017). https://doi.org/10.1136/ bmj.j4008

Strauss, A., Corbin, J.: Competing paradigms in qualitative research. Handb. Qual. Res. 2(273-285), 105 (1994)

Thomas, B., Abdulrouf, P., Alhail, M., Elkassem, W., Singh, R., Alsaad, D., McLay, J., Maclure, K., Paudyal, V., Stewart, D.: Incidence, nature and causes of medication errors in hospitalised patients in Middle Eastern countries: a systematic review. Int. J. Clin. Pharm. 38, 557-558 (2016)

Vaismoradi, M., Jones, J., Turunen, H., Snelgrove, S.: Theme development in qualitative content analysis and thematic analysis. J. Nurs. Edu. Pract. (2016). https://doi.org/10.5430/jnep.v6n5p100

Van den Bos, J., Rustagi, K., Gray, T., Halford, M., Ziemkiewicz, E., Shreve, J.: The \$17.1 billion problem: the annual cost of measurable medical errors. Health Aff. 30, 596-603 (2011)

Walsh, E.K., Hansen, C.R., Sahm, L.J., Kearney, P.M., Doherty, E., Bradley, C.P.: Economic impact of medication error: a systematic review. Pharmacoepidemiol. Drug Saf. 26, 481-497 (2017)

Wiegand, P.N., Nathwani, D., Wilcox, M.H., Stephens, J., Shelbaya, A., Haider, S.: Clinical and economic burden of Clostridium difficile infection in Europe: a systematic review of healthcare-facility-acquired infection. J. Hosp. Infect. 81, 1-14 (2012)

Publisher's Note Springer Nature remains neutral with regard to jurisdictional claims in published maps and institutional affiliations.

\section{Authors and Affiliations}

\section{Ehsan Ahsani-Estahbanati ${ }^{1} \cdot$ Leila Doshmangir $^{1,2}$ (D) $\cdot$ Behzad Najafi $^{3} \cdot$ Ali Akbari Sari $^{4}$. Vladimir Sergeevich Gordeev ${ }^{5,6}$}

1 Department of Health Policy \& Management, Tabriz Health Services Management Research Center, Iranian Center of Excellence in Health Management, School of Management \& Medical Informatics, Tabriz University of Medical Sciences, Daneshgah Street, 5166614711 Tabriz, Iran

2 Social Determinants of Health Research Center, Tabriz University of Medical Sciences, Tabriz, Iran

3 Department of Health Economics, School of Management \& Medical Informatics, Tabriz University of Medical Sciences, Tabriz, Iran

4 Department of Health Management and Economics, School of Public Health, Tehran University of Medical Sciences, Tehran, Iran

5 Wolfson Institute of Population Health, Barts and The London School of Medicine and Dentistry, Queen Mary University of London, London, UK

6 Department of Infectious Disease Epidemiology, London School of Hygiene \& Tropical Medicine, London, UK 